\title{
Factors affecting Human Immunodeficiency Virus/ Acquired Immune Deficiency Syndrome preventive behavior among pregnant women in ANC clinic in maternity hospital, Nepal
}

Sumi Amatya

From $16^{\text {th }}$ International Symposium on HIV and Emerging Infectious Diseases

Marseille, France. 24-26 March 2010

\section{Background}

To determine the factors affecting HIV preventive behaviors among pregnant women attending antenatal care clinic in Maternity hospital Kathmandu, Nepal.

\section{Methods}

A cross sectional study using questionnaire, among 150 pregnant women, ranging from 17 to 35 years of age, attending ANC clinic at Maternity hospital, Kathmandu, Nepal in 2005.

\section{Results}

No significant association between age and overall HIV preventive behavior. $(\mathrm{p}=0.866)$; and sexual HIV prevention $(\mathrm{p}=0.535)$ or asexual HIV preventive behavior. $(p=0.180)$. Significant association between occupational status and overall HIV preventive behavior $(\mathrm{p}=0.000)$; and sexual HIV preventive behavior $(\mathrm{p}=0.000)$. No significant association between occupational status and asexual HIV preventive behavior $(\mathrm{p}=0.058)$, but employed women showed higher tendency to have good asexual HIV preventive behavior than unemployed (14\% versus $4.3 \%$ ). Significant association between financial status and overall HIV preventive behavior $(\mathrm{p}=0.001)$; and sexual HIV preventive behavior $(\mathrm{p}=0.000)$. There is no significant association between levels of knowledge; perception of the respondents; having experience of STI and overall HIV preventive behavior.

\section{Discussion}

Study reveals that women have high perceptions of HIV susceptibility but have low efficacy. The pattern of sexual behavior cannot be separated from wider social and cultural influences. With majority of women with only primary level education, unemployment and at low income group, the socio-cultural influence is felt heavily in their sexual behavior such that despite having good knowledge and high HIV perception, women in this study still did not possess good HIV preventive practices. Hence, innovative social marketing HIV/STD prevention is needed for women to perceive benefits of prevention behaviors greater than the barriers to the behavior. Scale-up of HIV prevention programme requires inclusion of sex education and training on negotiation skills in a sexual relationship to women at school, community, workplaces, health post and hospital counseling services.

Published: 11 May 2010

doi:10.1186/1742-4690-7-S1-P3

Cite this article as: Amatya: Factors affecting Human Immunodeficiency Virus/Acquired Immune Deficiency Syndrome preventive behavior among pregnant women in ANC clinic in maternity hospital, Nepal. Retrovirology 2010 7(Suppl 1):P3.

Correspondence: amatyasumi@yahoo.com

London School of Hygiene and Tropical Medicine, London, UK 